\title{
Loop Quantum Gravity Vacuum with Nondegenerate Geometry ${ }^{\star}$
}

\author{
Tim KOSLOWSKI ${ }^{\dagger}$ and Hanno SAHLMANN ${ }^{\ddagger}$ \\ † Perimeter Institute for Theoretical Physics, Waterloo, Canada \\ E-mail: tkoslowski@perimeterinstitute.ca \\ ¥ APCTP, and Physics Department of POSTECH University, Pohang, Korea \\ E-mail: sahlmann@apctp.org
}

Received September 23, 2011, in final form May 03, 2012; Published online May 12, 2012

http://dx.doi.org/10.3842/SIGMA.2012.026

\begin{abstract}
In loop quantum gravity, states of the gravitational field turn out to be excitations over a vacuum state that is sharply peaked on a degenerate spatial geometry. While this vacuum is singled out as fundamental due to its invariance properties, it is also important to consider states that describe non-degenerate geometries. Such states have features of Bose condensate ground states. We discuss their construction for the Lie algebra as well as the Weyl algebra setting, and point out possible applications in effective field theory, Loop Quantum Cosmology, as well as further generalizations.
\end{abstract}

Key words: loop quantum gravity; representations, geometric condensate

2010 Mathematics Subject Classification: 83C45; 81R15; 46L30; 28C20

\section{Introduction}

Observables are quantum mechanically described by operators, and pure states by rays in a Hilbert space, so the mathematical problem of quantization is in part the representation theory of the underlying operator algebra. The importance of representation theory is in particular due to the fact that different irreducible representations of the same algebra may lead to physically distinct predictions. This can already be observed in the for physicists more familiar case of linear representations of the group SU(2). Thus, once a class of operator algebras describing the quantum observables of a system is found, its representation theory is a purely mathematical problem. Progress in this purely mathematical problem can however lead to significant physical insight.

Strong uniqueness theorems (referred to as F/LOST in the following) state that all regular irreducible representations with diffeomorphism invariant vacuum vector of the algebra that underlies Loop Quantum Gravity (LQG) are unitarily equivalent to the familiar AshtekarLewandowski (AL) representation. It turns out that the kinematic vacuum expectation values for all geometric operators, as well as their higher moments, vanish. The vacuum thus represents a degenerate spatial geometry, usually called "no geometry". The excitations over this vacuum are one dimensional 'flux-tubes' of non-degenerate geometry. It is thus not easy to approximate smooth geometries with them. That said, the AL ground state has the appealing feature that it is the "no geometry"-state, which is the natural vacuum for background independent quantum gravity, where any geometry is viewed as an excitation of the gravitational field.

However, the assumptions of invariance under spatial diffeomorphisms and irreducibility used in F/LOST are, while natural, not strictly necessary, at very least not if one is interested

\footnotetext{
*This paper is a contribution to the Special Issue "Loop Quantum Gravity and Cosmology". The full collection is available at http://www.emis.de/journals/SIGMA/LQGC.html
} 
in some sort of effective theory. Thus, dropping these assumptions is interesting and opens the door for new representations, in particular for representations with vacuum vectors that possess nonvanishing expectation values for spatial geometric operators. We review the explicit construction of such representations both for the Lie algebra (i.e. holonomy-flux algebra) as well as for a slight modification of the $C^{*}$-algebra (i.e. Weyl algebra) that underlies LQG.

Despite subtle technical differences, one finds in both cases the same basic features: There exists a smooth classical background geometry and discrete LQG excitations on top of the background geometry. This is analogous to states that describe a Bose condensate, where the field operator attains a nontrivial vacuum expectation value with quantum excitations around this VEV. One may thus view the vacuum vectors of the new representations as condensates of LQG. We conclude the paper by considering applications of the non degenerate representations in effective field theory, Wilsonian renormalization and discuss a possible connection with Loop Quantum Cosmology as well as the possibility of yet more general representations.

This review is focused on [15, 16, 22]. For other non-standard representations with different motivations see $[10,31]$. The technical underpinnings of the AL representation can be found in $[1,2]$. The F/LOST uniqueness result for the holonomy-flux algebra is based on $[23,24,26]$ and was further developed in [19,20], and together with the irreducibility result [25] resulted in [18]. The $C^{*}$-algebra was developed together with the corresponding irreducibility and uniqueness result in $[11,12,13]$. For an complementary approach to continuous geometries in LQG from the covariant perspective, see for example [21].

\section{Algebras underlying Loop Quantum Gravity}

LQG is a canonical quantization program for gravity, based on a formulation in terms of connection variables. The canonical pair to be quantized consists of an $\mathrm{SU}(2)$ connection $A$ and a densitized triad field $E$. These fields transform under the automorphisms of the $\mathrm{SU}(2)$ bundle. Upon choosing (local) trivializations, these bundle automorphisms split into gauge transformations $g$ and diffeomorphisms $\phi$,

$$
\begin{aligned}
& A \stackrel{g}{\longmapsto} \operatorname{Ad}_{g}(A)+g^{-1} d g, \quad A \stackrel{\phi}{\longmapsto} \phi_{*} A, \\
& E \stackrel{g}{\longmapsto} \operatorname{Ad}_{g}(E)=: g \triangleright E, \quad E \stackrel{\phi}{\longmapsto} \phi_{*} E=: \phi \triangleright E,
\end{aligned}
$$

where $\mathrm{Ad}$ is the adjoint action of $\mathrm{SU}(2)$ on $\mathrm{su}(2)$, and the star signifies push-forward. The automorphism group is then the semi-direct product of those subgroups. Poisson brackets are given by

$$
\left\{A_{a}^{I}(x), E_{J}^{b}(y)\right\}=8 \pi G \beta \delta_{J}^{I} \delta(x, y) .
$$

Point fields are usually too singular to give well defined operators in the quantum theory, so a Poisson algebra of smoother functions on phase space has to be chosen. It is characteristic for LQG that one considers holonomies

$$
h_{e}[A]=\mathcal{P} \exp \int_{e} A,
$$

or more generally, functions of such holonomies as basic functionals of the connection. To be precise, consider functionals of the form

$$
f[A] \equiv F\left(h_{e_{1}}[A], h_{e_{2}}[A], \ldots, h_{e_{n}}[A]\right)
$$

for a finite number of paths $e_{1}, \ldots, e_{n}$ forming a graph, and a function $F$ on $n$ copies of $\mathrm{SU}(2)$. Such functionals are also called cylindrical functions, and the algebra they generate is denoted Cyl. Cyl carries a natural norm induced by the sup norm for continuous functions 
on $\mathrm{SU}(2)$. Closure under this norm gives a $\mathrm{C}^{*}$ algebra $\overline{\mathrm{Cyl}}$ which is isomorphic to $C(\overline{\mathcal{A}})$, the algebra of continuous functions on a set of distributional connections, the so-called generalized connections. Diffeomorphisms and gauge transformations act as

$$
\begin{aligned}
& (g \triangleright f)[A]=F\left(g\left(e_{1}(0)\right)^{-1} h_{e_{1}} g\left(e_{1}(1), g\left(e_{2}(0)\right)^{-1} h_{e_{2}} g\left(e_{2}(1)\right), \ldots\right)\right), \\
& (\phi \triangleright f)[A]=F\left(h_{\phi\left(e_{1}\right)}[A], h_{\phi\left(e_{2}\right)}[A], \ldots\right) .
\end{aligned}
$$

For the triad fields $E$ on the other hand, the choice of functional is not so clear. One natural choice is its flux through surfaces $S$ :

$$
E_{S, r}[E]=\int_{S} * E_{I} r^{I}
$$

where $r$ is a smooth function taking values in $\operatorname{su}(2)^{*}$ and vanishing on $\partial S$, and $* E$ is the two-form $E^{a} \epsilon_{a b c} \mathrm{~d} x^{b} \wedge \mathrm{d} x^{c}$. Another natural choice is to use the imaginary exponentials of those fluxes,

$$
w_{S, r}=e^{i E_{S, r}} .
$$

These two possibilities lead to slightly different algebras which we will discuss next.

\subsection{Holonomy-flux algebra}

We start with the commutative algebra $\mathrm{Cyl}$ and add elements $E_{S, r}$ for surfaces $S$ and maps $r$ to $\mathrm{su}(2)^{*}$ as above. These elements have non-trivial commutation relations with the elements of Cyl and among each other. To begin with,

$$
\left[E_{S, r}, f\right]=8 \pi \beta l_{P}^{2} X_{S, r}[f]
$$

which are nothing but the canonical commutation relations. $X_{S, r}[f]$ is an element of Cyl obtained from $f$ by action of a certain derivation $X$. Then we have

$$
\left[f,\left[E_{S_{1}, r_{1}}, E_{S_{2}, r_{2}}\right]\right]=\left(8 \pi \beta l_{P}^{2}\right)^{2}\left[X_{S_{1}, r_{1}}, X_{S_{2}, r_{2}}\right][f]
$$

and similar higher order relations. These are consequences of (1) and the requirement that the commutator in the resulting algebra satisfy the Jacobi identity.

There are also relations, concerning linearity in the $r_{i}$ and related properties, which we will not discuss in detail here. Finally, the algebra becomes a *-algebra by setting $E_{S, r}^{*}=E_{S, r}$ for real $r$. We will denote this algebra with $\mathfrak{H} \mathfrak{F}$. Diffeomorphisms and gauge transformations act as automorphisms of $\mathfrak{H} \mathfrak{F}$,

$$
\left(g \triangleright E_{S, r}\right)=E_{S, r \circ A_{g}-1}, \quad\left(\phi \triangleright E_{S, r}\right)=E_{\phi(S), \phi_{*} r} .
$$

A more precise definition of $\mathfrak{H F}$ can be found for example in [18].

\section{$2.2 \quad C^{*}$-algebra (Weyl algebra)}

Given a $C^{*}$-algebra ${ }^{1} \mathfrak{A}$ and a state ${ }^{2} \omega: \mathfrak{A} \rightarrow \mathbb{C}$, one can construct a Hilbert space and a continuous $*$-representation of the $C^{*}$-algebra through the GNS construction, which involves the following steps:

\footnotetext{
${ }^{1} \mathrm{~A} C^{*}$-algebra over $\mathbb{C}$ is an algebra $\mathfrak{A}$ that is also Banach space w.r.t. a norm $\|\cdot\|: \mathfrak{A} \rightarrow \mathbb{R}_{o}^{+}$and an involution $*: \mathfrak{A} \rightarrow \mathfrak{A}$, s.t. $\left\|a^{*} a\right\|=\|a\|^{2}$ for all $a \in \mathfrak{A}$.

${ }^{2}$ A state is a continuous linear functional satisfying positivity $\omega\left(a^{*} a\right) \geq 0$ for all $a \in \mathfrak{A}$, as well as satisfying $\omega(1)=1$ if $\mathfrak{A}$ is unital.
} 
1. Consider the quotient of $\mathcal{H}_{\omega}^{o}=\mathfrak{A} / \mathcal{I}_{\omega}$, where $\mathcal{I}_{\omega}:\left\{a \in \mathfrak{A}: \omega\left(a^{*} a\right)=0\right\}$ denotes the Gel'fand ideal.

2. Quotienting by $\mathcal{I}_{\omega}$ defines the map $\eta_{\omega}: \mathfrak{A} \rightarrow \mathcal{H}_{\omega}^{o}$, which defines a representation $\pi_{\omega}$ of $\mathfrak{A}$ on $\mathcal{H}_{\omega}^{o}$ by $\pi_{\omega}(a): \eta_{\omega}(b) \mapsto \eta_{\omega}(a b)$ for all $a, b \in \mathfrak{A}$.

3. Define the inner product $\langle\cdot, \cdot\rangle_{\omega}: \mathcal{H}_{\omega}^{o} \times \mathcal{H}_{\omega}^{o} \rightarrow \mathbb{C}$ by $\left\langle\eta_{\omega}(a), \eta_{\omega}(b)\right\rangle_{\omega}:=\omega\left(a^{*} b\right)$ and complete $\mathcal{H}_{\omega}^{o}$ to a Hilbert space $\mathcal{H}_{\omega}$ in this inner product.

4. Extend the representation $\pi_{\omega}$ by continuity from $\mathcal{H}_{\omega}^{o}$ to $\mathcal{H}_{\omega}$.

If $\mathfrak{A}$ has a unit then $\eta_{\omega}(1)$ is a cyclic vector $\Omega_{\omega}$; moreover any representation $(\pi, \mathcal{H})$ of $\mathfrak{A}$ with cyclic $^{3}$ normalized vector $v \in \mathcal{H}$ defines a state $\omega_{v}$ defines by $\omega_{v}(a):=\langle v, \pi(a) v\rangle_{\mathcal{H}}$ and the GNS-representation constructed from $\omega_{v}$ is unitarily equivalent to $(\pi, \mathcal{H})$, so there is a one to one correspondence between cyclic representations and states.

To apply this framework to LQG, Fleischhack considers a class of $C^{*}$-algebras that naturally includes the Weyl algebra of LQG. The class of algebras he considers is constructed from structure data given by a base manifold $\mathbb{X}$, a compact gauge group $\mathbb{G}$, a groupoid $\mathcal{P}$ of paths in $\mathbb{X}$ of a certain smoothness class, a subset $\mathcal{S}$ of the quasi-surfaces in $\mathbb{X}$ and for each $S \in \mathcal{S}$ a subset $\Sigma(S)$ of their intersection functions and a subset $\Delta(S)$ of functions $\mathbb{X} \rightarrow \mathbb{G}$, as well as a subset $\mathcal{D}$ of the graphomorphisms that leave $\mathcal{S}$ invariant and act covariantly on $\Sigma$ and $\Delta$. Fleischhack then uses additional restrictions (as optimal data) on the structure data that ensure that the constructions of the F/LOST theorem hold. This abstract structure data can be used to construct a generalization of the algebra that underlies LQG; we will now illustrate this construction.

1. The configuration space of LQG consists of generalized connections, which are morphisms from the path groupoid $\mathcal{P}$ to the gauge group $\mathbb{G}$. This is a generalization of the morphisms defined by gauge field configurations given by $A: e \in \mathcal{P} \mapsto h_{e}(A) \in \mathbb{G}$, where $h_{e}(A)$ denotes the parallel transport along $e$. Using the partial order among graphs $\gamma$ (as finite subgroupoids $\mathcal{P}_{\gamma}$ of the path groupoid $\mathcal{P}$ ) given by the subgraph relation, one can equip the space $\mathcal{A}$ of the morphisms $A$ with the Tychonov topology inherited from the compactness of $\mathbb{G}$. The algebra $C(\mathcal{A})$ of continuous functions in this topology is the $C^{*}$-completion of continuous functions on morphisms $\mathcal{A}_{\gamma} \ni A_{\gamma}: \mathcal{P}_{\gamma} \rightarrow \mathbb{G}$. Functions that are determined by the dependence on the image of a finite number of elements of $\mathcal{P}$ are referred to as cylindrical functions; these are dense in $C(\mathcal{A})$.

2. Diffeomorphisms $\phi \in \mathcal{D}$ act by their pull-backs, so $\phi$ maps $A: e \mapsto h_{e}(A)$ to $\phi \triangleright A$ : $e \mapsto h_{\phi(e)}(A)$. Gauge transformations $\Lambda: \mathbb{X} \rightarrow \mathbb{G}$ act on $A: e \mapsto h_{e}(A)$ by $\Lambda \triangleright A: e \mapsto$ $\Lambda^{-1}(e(0)) h_{e}(A) \Lambda(e(1))$, where $e(0), e(1)$ denote the initial resp. final point of $e$. Both sets of transformations act as natural transformations of the functors $A$.

3. The (uniform) Ashtekar-Isham-Lewandowski measure $d \mu_{A I L}$ on $\mathcal{A}$ is defined as the measure whose push-forward under the projections from $\mathcal{A}$ onto $\mathcal{A}_{\gamma}$ coincides with the product of Haar measures of $\mathbb{G}$ on each edge $e \in \gamma$. This allows us to construct a Hilbert space $\mathcal{H}_{A L}=L^{2}\left(\mathcal{A}, d \mu_{A I L}\right)$ on which the algebra of cylindrical functions (and its $C^{*}$-completion) act as multiplication operators. Moreover, since $d \mu_{A L}$ is invariant under the pull-back action of diffeomorphisms and gauge-transformations, one can define unitary operators on $\mathcal{H}_{A L}$ acting as $U_{\phi}: f(A) \mapsto f(\phi \triangleright A)$ and $U_{\Lambda}: f(A) \mapsto f(\Lambda \triangleright A)$ for $\phi \in \mathcal{D}$ and $\Lambda \in \Delta$.

4. The definition of the exponentiated momentum (flux) operators is subtle: An oriented surface $S$ defines an intersection function $\sigma(S, e)$ vanishing for edges $e$ completely within or completely outside $S$ and \pm 1 for edges beginning resp. ending above resp. below $S$.

\footnotetext{
${ }^{3} v$ is cyclic if $\{\pi(a) v\}_{a \in \mathfrak{A}}$ is dense in $\mathcal{H}$.
} 
Moreover, if both $S$ and $e$ are restricted to the piecewise analytic category then every edge $e=e_{1} \circ \cdots \circ e_{n}$ can be finitely decomposed into $S$-admissible pieces $e_{i}$ that are either completely inside, outside or beginning or ending on $S$. Let $\Lambda \in \Delta(S)$ then we define the translation $\Theta_{\Lambda, S, \sigma}$ on $\mathcal{A}$ by $\Theta_{\Lambda, S, \sigma} A: e_{i} \mapsto \Lambda^{-\sigma\left(S, e_{i}\right)}\left(e_{i}(0)\right) h_{e}(A) \Lambda^{\sigma_{S, e_{i}}}\left(e_{i}(1)\right)$, where the pieces $e_{i}$ are assumed to be $S$-admissible. We can now define the Weyl operators corresponding to exponentiated flux operators as the pull-backs under these translations: $w_{\Lambda, S, \sigma}:=\Theta_{\Lambda, S, \sigma}^{*}$. It turns out that these Weyl operators leave the Ashtekar-Isham-Lewandowski measure invariant and can thus be extended to unitary operators on $\mathcal{H}_{A L}$. There is however and additional subtlety: Products of Weyl operators through natural surfaces require us to include lower dimensional "quasisurfaces" $S$ and non-natural intersections functions $\sigma$ in the group $\mathcal{W}$ generated by the natural Weyl operators $w_{\Lambda, S, \sigma}$.

5 . With these preparations we define the $*$-algebra $\mathfrak{A}_{o}$ as the algebra of finite sums of ordered products $a=\sum_{i=1}^{n} f_{i} \circ w_{i}$, where $f_{i} \in C(\mathcal{A})$ and $w_{i} \in \mathcal{W}$. Using the the action of $C(\mathcal{A})$ as multiplication operators and $\mathcal{W}$ as pull-backs under translations on $\mathcal{H}_{A L}$ we have a canonical representation of $\mathfrak{A}_{o}$ as bounded operators on $\mathcal{H}_{A L}$. This allows us to define the completion $\mathfrak{A}$ as the smallest $C^{*}$-algebra in $\mathcal{B}\left(\mathcal{H}_{A L}\right)$ that contains $\mathfrak{A}_{o}$.

The operators $f \in C(\mathcal{A}) \subset \mathfrak{A}$ are the configuration operators and the operators $w \in \mathcal{W} \subset \mathfrak{A}$ are the exponentiated momentum operators of LQG and the diffeomorphisms and gauge transformations have same action as in LQG. To obtain the usual (unbounded) flux operators, one has to take the derivative at the unit element of $\mathcal{W}$, which imposes a continuity condition on the representation of $\mathfrak{A}$. This justifies calling $\mathfrak{A}_{o}$ the Weyl algebra underlying LQG.

\section{$2.3 \quad$ F/LOST representation theorem}

The AL-representations that we have described above are fundamental for LQG in a specific sense: They are the unique representations with certain natural properties. This is the content of the uniqueness results $[13,18,20,24,26]$. Roughly speaking, the properties that specify the representations uniquely are the following:

1. Cyclicity. There exists a vector $\Omega$ in the representation Hilbert space such that application of all the operators of the algebra creates a set of vectors dense in the Hilbert space.

2. Invariance. The cyclic vector $\Omega$ is invariant under diffeomorphisms.

We should point out that in the case of the holonomy-flux algebra, the requirement of cyclicity presupposes that $\Omega$ is in the domain of all the operators representing $\mathfrak{H} \mathfrak{F}$, and it implies that there is a common dense domain for all of them. In the case of the Weyl-like algebra $\mathfrak{A}$ one requires additionally

3. Regularity. The representation of the Weyl elements $w \in \mathcal{W}$ has to be continuous in the smearing function.

Cyclicity is natural in that it follows from irreducibility. The cyclic representations are thus the simple building blocks out of which one can build more complicated representations. Another way to motivate cyclicity is to view it as a replacement of the lowest energy property of ground states in the absence of a good notion of energy.

Invariance is a natural requirement given the fact that the constraints of canonical gravity in the Ashtekar-Barbero variables require, among other things, invariance under gauge transformations and spatial diffeomorphisms. In fact, the AL vacuum turns out to be a solution of all the constraints of LQG. Moreover cyclicity and invariance together imply that gauge transformations and spatial diffeomorphisms are implemented by unitary operators. This in turn greatly facilitates finding general solutions to the constraints via group averaging (see for example [5]). 
Regularity insures that the fluxes themselves are available in the representation, thus making it possible to obtain geometric operators and facilitate quantization of the Hamiltonian constraint.

It should be noted that while the conditions above are the main ones that lead to uniqueness, there are further technical conditions in the uniqueness theorems. For details we refer to the original literature. Perhaps more importantly, we want to emphasize that while the requirements are natural, they are not absolutely necessary. This is due to the fact that the algebras and representations under consideration are kinematic by nature. The algebra elements are generically not observables, and the states generically do not solve the constraints. Rather, these representations are a departure point for constructing physical states and observables. If other ways to achieve this can be found, they may be equally viable, or at least interesting in certain contexts. It is in this spirit that we will drop the invariance condition and construct representations with ground states that describe non-degenerate geometries in the next section.

\section{Nondegenerate vacua and representations}

The cyclic state of the AL-representation is an eigenstate of all the flux operators (and, more generally, of all geometric operators) with eigenvalue zero. Conversely, it maximizes quantum mechanical uncertainty in the holonomies in a precise technical sense. Thus this state treats the canonical variables quite asymmetrically. On a fundamental level, some justification for this is provided by the uniqueness theorems that were briefly described above. But in order to more effectively describe non-degenerate geometries in the quantum theory, there have long been efforts to find states that divide uncertainty more evenly, and give the flux operators nonzero expectation values. One strategy is to seek states with such properties in $\mathcal{H}_{\mathrm{AL}}$, leading to weave states [6] and coherent states [7, 8, 9, 14, 27, 28]. These states achieve the stated goals for a finite number of degrees of freedom, but there are difficulties to extend this to infinitely many degrees of freedom. A quick way to see the source of these difficulties is the following consideration $^{4}$. Instead of trying to construct a smoothly peaked function, let us first consider characteristic functions

$$
\chi_{M}(A):= \begin{cases}1, & A \in M, \\ 0, & \text { otherwise }\end{cases}
$$

for some subset $M \subset \overline{\mathcal{A}}$. Pick a neighborhood $M_{g_{0}}$ around a point $g_{0}$ in $\mathrm{SU}(2)$ and let $M_{e, g_{0}}=$ $\left\{A \in \overline{\mathcal{A}}: h_{e}(A) \in M_{g_{0}}\right\}$ for some edge $e$. Then it is easy to show that

$$
\left\|\chi_{M_{e, g_{0}}}\right\|=\operatorname{Vol}\left(M_{g_{0}}\right)
$$

where Vol denotes the volume on the group. This shows that the norm of this characteristic function can get small, despite the fact that the set $M_{e, g_{0}}$ is huge. Restricting further holonomies to the neighborhood $M_{g_{0}}$ results in

$$
\left\|\chi_{M_{e_{1}, e_{2}, \ldots, e_{n}, g_{0}}}\right\|=\operatorname{Vol}\left(M_{g_{0}}\right)^{n} .
$$

From this it is clear that to obtain something that is peaked on infinitely many edges, one has to rescale the characteristic functions and take a limit, and that this limit can not be a function anymore.

Indeed there exist objects that have the properties of Gaussians outside of $\mathcal{H}_{\mathrm{AL}}$, namely Gaussian measures on $\overline{\mathcal{A}}$. Measures on $\overline{\mathcal{A}}$ are generally given by families of measures on cylindrical

\footnotetext{
${ }^{4}$ It was developed together with M. Varadarajan.
} 
subspaces, fulfilling certain consistency conditions [1, 2]. For Gaussian measures [4, 24, 27, 30], the family has the form

$$
\mathrm{d} \mu_{\gamma}=e^{-G_{\gamma}{ }_{e e^{\prime}}^{I J} X_{I}^{e} X_{J}^{e^{\prime}}} \delta_{z}^{\gamma} \mathrm{d} \mu_{\mathrm{Haar}}
$$

where $G_{\gamma}$ is a graph metric, the $X$ represent invariant vector fields acting on the variables corresponding to the various edges of the graph $\gamma$, and the delta-function is $\delta^{\gamma}$ is peaked at a point $z$ in the complexification of $\mathrm{SU}(2)^{|\gamma|}$. Consistency of the family translates into conditions on the family of $G_{\gamma}$, and some solutions are known [4]. The problem with these measures is that, while they furnish new representations for $\mathrm{Cyl}$, they generally do not support representations of the fluxes [24]. The reason is that to obtain symmetric operators, the fluxes have to acquire a measure dependent divergence term,

$$
\pi\left(E_{S, f}\right)=X_{S, f}+\frac{i}{2} \operatorname{div}_{\mu}(S, f) .
$$

$\operatorname{div}_{\mu}(S, f)$ is formally an operator which preserves the commutation relations and compensates for the failure of $X$ to be symmetric, i.e., $i \operatorname{div}_{\mu}(S, f)=X_{S, f}^{\dagger}-X_{S, f}$. For the known measures, the divergence term is so singular that a representation of the fluxes by symmetric operators simply does not exist [24]. Thus we do not know any states that divide uncertainty more evenly between more than a finite number of canonical pairs. But while we do not know how to broaden the delta-function-like peak of the AL ground state it turns out that it is possible to shift this peak to non-degenerate geometries. This is already a big step in and of itself, because the resulting representations describe excitations over a geometric background. But it may only be the first step towards some form of coherent states that more accurately represent the observed geometric condensate that we classically describe as a space-time geometry. The states that we review more precisely below, contain a condensate of spatial geometry, but the extrinsic curvature of the spatial slice, and hence the space-time geometry is highly fluctuating and quantum mechanical in these states. We will now turn to a more detailed description.

\subsection{Representations of the holonomy-flux algebra with nondegenerate background geometry}

Now we will discuss the representations of $\mathfrak{H} \mathfrak{F}$ with nondegenerate background geometry [16, 22]. Let $E^{(0)}$ be a classical triad field. Then we can define a new representation $\pi^{\prime}$ on the AL Hilbert space $\mathcal{H}_{\mathrm{AL}}$, by changing the action of the fluxes:

$$
\pi^{\prime}\left(E_{S, r}\right)=X_{S, r}+E_{S, r}^{(0)} \mathrm{id}, \quad \text { with } \quad E_{S, r}^{(0)}=\int_{S} * E^{(0) I} r_{I}
$$

It is easily checked that this gives another representation of the algebra $\mathfrak{H F}$. We list some elementary properties.

1. The spectra of the fluxes have changed. If $\lambda$ is an eigenvalue of $X_{S, f}$ then $\lambda+E_{S, f}^{(0)}$ is an eigenvalue of $\pi^{\prime}\left(E_{s, f}\right)$. In particular, the AL vacuum $|\varnothing\rangle$ is now an eigenstate of $\pi^{\prime}\left(E_{s, f}\right)$ with eigenvalue $E_{S, f}^{(0)}$, which is non-vanishing in general.

2. The new representation is still cyclic, with $|\varnothing\rangle$ as cyclic vector.

3. The new representation is unitarily inequivalent to the AL representation.

4. The standard kinematic representation can be viewed as a special case of the new one, for $E^{(0)}=0$. 
1 is immediate since the new term is proportional to the identity and hence all states are its eigenstates. 2 can be shown by making use of the fact that the unit operator is part of the algebra. It can be used to remove the new terms in (2) and show that old and new representation generate the same dense subspace upon acting on $|\varnothing\rangle$. As for 3, the new representation is unitarily inequivalent, because a unitary transformation can not change the spectra. 4 is obvious.

To better interpret the new representations, it is very useful to consider the action of operators corresponding to geometric measures such as area and volume. In the AL-representation, these are defined by giving a regularized expression in terms of the elementary variables, quantizing this regularized expression, and then taking the cutoff to zero in the quantum theory [3, 17]. A priori, it is not clear if this strategy still works in the new representation since, due to the extra term in (2), the regularized operators contain additional terms, the number of which tends to infinity as the regulator is removed. It turns out, however, that all goes well.

Proposition 1 ([22]). The standard regularization and quantization procedures for area and volume operators can be applied to the new representations and lead to well defined operators. We find

$$
V_{R}=V_{R}^{\mathrm{vac}}+V_{R}^{(0)} \mathrm{id}, \quad A_{S}=A_{S}^{\mathrm{vac}}+A_{S}^{(0)} \mathrm{id}
$$

with $V^{\mathrm{vac}}, A^{\mathrm{vac}}$ the geometric operators in the vacuum representation, and $V_{R}^{(0)}, A_{S}^{(0)}$ the classical values in the background geometry.

This shows clearly that the new representations contain a background geometry given by the background field $E^{(0)}$.

We turn next to the gauge symmetries of LQG: The operators implementing diffeomorphisms and gauge transformations in the standard representation are still well defined and unitary in the new representation, but it can be easily checked that they do not implement the algebraautomorphisms anymore. For example

$$
U_{\phi} \pi^{\prime}\left(E_{S, f}\right) U_{\phi^{-1}}=U_{\phi}\left(X_{S, f}+E_{S, f}^{(0)} \mathbb{I}\right) U_{\phi^{-1}}=X_{\phi(S), \phi_{*} f}+E_{S, f}^{(0)} \mathbb{I} \neq \pi^{\prime}\left(\phi \triangleright E_{S, f}\right),
$$

by virtue of the fact that the $U_{\phi}$ act on the $X_{S, f}$ in the standard way, but commute with the new c-number term. In other words, the problem is that the $U_{\phi}$ do not change the background geometry.

This can be remedied by going over to a large direct sum of Hilbert spaces. For a given background $E^{(0)}$ we consider

$$
\mathcal{H}_{G\left(E^{(0)}\right)}:=\bigoplus_{\widetilde{E}^{(0)} \in G\left(E^{(0)}\right)} \mathcal{H}_{\widetilde{E}^{(0)}}
$$

where $G\left(E^{(0)}\right)$ is the equivalence class of $E^{(0)}$ with respect to diffeomorphisms and gauge transformations. The Hilbert space is thus effectively labeled by a spatial background metric modulo diffeomorphisms. We will write $\left|T, \widetilde{E}^{(0)}\right\rangle$ for the cylindrical state $T$ in the (new) representation with background geometry $\widetilde{E}^{(0)}$ in the above Hilbert space. Thus we have

$$
\left\langle T, E^{(0)} \mid T^{\prime}, E^{\prime(0)}\right\rangle=\left\langle T \mid T^{\prime}\right\rangle \delta_{E^{(0)}, E^{\prime(0)}},
$$

where the first scalar product on the right hand side is just the one in the vacuum representation. The Hilbert space $\mathcal{H}_{G\left(E^{(0)}\right)}$ carries a representation of $\mathfrak{H} \mathfrak{F}$, which is simply the direct sum of the representations on the individual spaces. But now one can implement the diffeomorphisms and gauge transformations unitarily, by setting [16, 22]

$$
U_{\phi}\left|T, E^{(0)}\right\rangle=\left|\phi \triangleright T, \phi \triangleright E^{(0)}\right\rangle, \quad U_{g}\left|T, E^{(0)}\right\rangle=\left|g \triangleright T, g \triangleright E^{(0)}\right\rangle .
$$


It is easy to show that with this definition, the transformations are also correctly implemented on the operators. A straightforward calculation shows that not only the groups of gauge transformations and spatial diffeomorphisms are correctly implemented, but also their direct product.

The unitary implementation of the symmetries can now be used to find invariant states and Hilbert spaces by group averaging, just as has been done for the vacuum representation [3]. Let us consider the case of diffeomorphisms as example. We will call a diffeomorphism $\phi$ a symmetry of a triad $E^{(0)}$, if $\phi$ leaves $E$ invariant, $\phi \triangleright E^{(0)}=E^{(0)}$. Then

1. Let Diff be the group of all diffeomorphisms.

2. Let $\operatorname{Diff}_{\left(\alpha, E^{(0)}\right)}$ be the group of diffeomorphisms that are symmetries of $E^{(0)}$ and map the graph $\alpha$ onto itself.

3. Let TDiff $\left(\alpha, E^{(0)}\right)$ be the group of diffeomorphisms that are symmetries of $E^{(0)}$ and map each edge of the graph $\alpha$ onto itself. Note: Again, if $E^{(0)}=0$ these are just the diffeomorphisms mapping each edge of $\alpha$ onto itself, denoted as $\operatorname{TDiff}_{\alpha}$ in [3].

4. Let $\operatorname{Symm}_{\left(\alpha, E^{(0)}\right)}$ be the quotient $\operatorname{Diff}_{\left(\alpha, E^{(0)}\right)} / \operatorname{TDiff}_{\left(\alpha, E^{(0)}\right)}$.

Note that these definitions closely follow [3]. Let now $T_{\alpha}$ be a cylindrical function that depends on the holonomies of all the edges of $\alpha$ in a non-trivial way. We can then define the linear form $\left(T_{\alpha}, E^{(0)} \mid\right.$ on $\mathcal{H}_{G\left(E^{(0)}\right)}$ as follows:

$$
\left(T_{\alpha}, E^{(0)}\left|T_{\beta}, E^{\prime(0)}\right\rangle:=\sum_{G(\phi) \in \text { Diff } / \text { Diff }_{\left(\alpha, E^{(0)}\right)}}\left\langle T_{\alpha}, E^{(0)}\left|P_{\left(\alpha, E^{(0)}\right)} U_{\phi}^{\dagger}\right| T_{\beta}, E^{\prime(0)}\right\rangle,\right.
$$

where the projection $P_{\left(\alpha, E^{(0)}\right)}$ is defined as

$$
P_{\left(\alpha, E^{(0)}\right)}\left|T_{\alpha}, E^{(0)}\right\rangle:=\frac{1}{\left|\operatorname{Symm}_{\left(\alpha, E^{(0)}\right)}\right|} \sum_{G(\phi) \in \operatorname{Symm}_{\left(\alpha, E^{(0)}\right)}} U_{\phi}\left|T_{\alpha}, E^{(0)}\right\rangle .
$$

Now it is easy to show that

Proposition 2. The linear functionals $\left(T_{\alpha}, E^{(0)} \mid\right.$ are well defined, finite, and diffeomorphism invariant,

$$
\left(T_{\alpha}, E^{(0)} \mid \circ U_{\phi}=\left(T_{\alpha}, E^{(0)} \mid \quad \text { for all } \phi \in\right. \text { Diff . }\right.
$$

Similar results can be obtained for gauge transformations and, taking the semidirect product of diffeomorphisms and gauge transformations, for bundle automorphisms. One thereby obtains the automorphism invariant Hilbert space $\mathcal{H}_{\text {aut }}$. Thus the quantum kinematics can be developed to exactly the same point for the new representations as for the AL representation. What is more, the latter appears simply as a special case of the constructions we sketched above.

We should stress that, while $H_{G\left(E^{(0)}\right)}$ is very large, this is partially remedied by the group averaging. Vectors $\left|1, E^{(0)}\right\rangle,\left|1, E^{\prime(0)}\right\rangle \in H_{G\left(E^{(0)}\right)}$ are mapped onto the same vector in $\mathcal{H}_{\text {aut }}$. More generally

$$
\left(f, \phi \triangleright E \mid=\left(\phi^{-1} \triangleright f, E \mid .\right.\right.
$$

We should also say that it seems that there is no problem with operators on $\mathcal{H}_{\text {aut }}$. As an example consider the operator $V$ for the volume of the entire spatial slice. Also in the new representations, it commutes with all automorphisms. It thus defines an operator on $\mathcal{H}_{\text {aut }}$. Moreover, this operator acts in precisely the way one would expect. If $f$ is an eigenstate of $V^{\mathrm{vac}}$, with eigenvalue $\lambda$, then $\left.\mid f, E^{(0)}\right)$ is an eigenvector of $V$ with eigenvalue $\lambda+V^{(0)}$. 


\subsection{Nondegenerate representations of the Weyl algebra}

Let us comment on the structure of the Weyl algebra of LQG, before we construct nondegenerate representations of a slight modification of $\mathfrak{A}_{o}$. We have a compact configuration space $\mathcal{A}$ and a group $\mathcal{W}$ of transformations acting on this space. Given such data one can build the a $*$-algebra of finite sums of the form $a=\sum_{i=1}^{n} f_{i} \circ w_{i}$, where $f_{i} \in C(\mathcal{A})$ and $w_{i} \in \mathcal{W}$. The multiplication is given by linearity and $\left(f_{1} \circ w_{1}\right)\left(f_{2} \circ w_{2}\right)=f_{1} \alpha_{w_{1}}\left(f_{2}\right) \circ w_{1} w_{2}$ and the involution is given by antilinearity and $(f \circ w)^{*}=\alpha_{w^{-1}}(\bar{f}) \circ w^{-1}$, where $\alpha_{w}$ denotes the action of the Weyl operators as pull-backs. Given a normalized $\mathcal{W}$-invariant measure $d \mu$ on $\mathcal{A}$, one finds by direct computation that

$$
\omega_{o}(f \circ w):=\int d \mu(A) f(A)
$$

is a bounded positive linear functional on $\mathfrak{A}_{o}$ and can thus be used for the GNS construction, which yields the standard Hilbert space representation of LQG. The definition (4) can be generalized in various ways; let us e.g. assume we have a character ${ }^{5} \chi$ of the group $\mathcal{W}$, then we can consider the functional obtained by the linear extension of

$$
\omega_{\chi}(f \circ w):=\chi(w) \int d \mu(A) f(A) .
$$

One can prove positivity by confirming $\omega\left(a^{*} a\right)=\int d \mu(A)\left|\sum_{i=1}^{n} f_{i}(A)\right|^{2} \geq 0$. It will soon be important that this only requires that $d \mu$ is invariant under $\mathcal{W}$, but not that the action $\alpha$. of $\mathcal{W}$ on $C(\mathcal{A})$ is by pull-backs.

To apply the definition (5), we have to find a character of the noncommutative group of Weyl operators $\mathcal{W}$, which seems hopeless because $\mathcal{W}$ is noncommutative and infinite dimensional. We thus consider a slightly different group $\mathcal{W}^{\prime}$. For this we fix a maximal commutative subgroup $\tau(x)$ of $S U(2)$ at each point in $x \in \mathbb{X}$ and for each surface $S$ consider only the Weyl operators $w_{\Lambda_{\tau}, S, \sigma}=w_{\Lambda, S, \sigma}$ where $\Lambda(x)$ takes values in $\tau(x)$. This group is too small to construct LQG. To be able to construct a version of LQG, we consider the standard LQG area operators $\hat{A}(S)$ for each surface $S$. We now use denseness of the gauge-variant spin network functions $T$ in $C(\mathcal{A})$ and consider the action of exponentiated area operators $w_{\lambda, S, A}=e^{i \lambda \hat{A}(S)}$, which act by multiplication of a phase $e^{6} e^{i \lambda \phi(S, T)}$ on the gauge-variant spin network functions. We now observe that the action of the area operators leaves $d \mu_{A I L}$ invariant. This is easily seem by expanding an element $f$ of $C(\mathcal{A})$ in terms of spin network functions $f=f_{o} 1+\left(f-f_{o} 1\right)$ and noticing that $\int d \mu(A) f(A)=f_{o}$ and that the phase of the constant spin network function 1 vanishes for all area operators. As in the case of the standard Weyl algebra, we have to include quasi-surfaces in the group $\mathcal{W}^{\prime}$ that is generated by the Weyl operators $w_{\lambda_{\tau}, S, \sigma}$ and $w_{\lambda, S, A}$. It turns out that all group elements of $\mathcal{W}^{\prime}$ leave $d \mu_{A I L}$ invariant and commute mutually. We denote the modified Weyl algebra by $\mathfrak{A}_{o}^{\prime}$.

To construct a character $\chi$ on $\mathcal{W}^{\prime}$, we use a densitized inverse triad $E$ describing a smooth classical geometry on $\mathbb{X}$ and define for 2-dimensional natural surfaces $S$

$$
\chi_{E}\left(w_{\Lambda_{\tau}, S, \sigma_{\text {nat. }}}\right)=e^{i \int_{S} \Lambda_{\tau} \cdot E}, \quad \chi_{E}\left(w_{\lambda, S, A}\right)=e^{i \lambda A_{E}(S)},
$$

where $A_{E}(S)$ denotes the classical area of $S$ calculated from $E$ and where

$$
\left(\Lambda_{\tau} . E\right)(x)=\Lambda(x) \tau_{I}(x) E^{I}(x)
$$

\footnotetext{
${ }^{5}$ A character of a group $G$ is a continuous morphism from $G$ to $U(1)$.

${ }^{6}$ Notice that the action of the area operator on gauge-invariant spin-network functions is much more complicated, because one has to choose a recoupling basis. It follows that a gauge-invariant spin network containing vertices of valence four or more can not be a simultaneous eigenstate of all area operators.
} 
denotes a 2-form. All other elements of $\mathcal{W}^{\prime}$ are mapped to 1 . Using $\chi_{E}$ in the state definition (5) to define the state $\omega_{E}$, we see that we can construct a GNS representation of $\mathfrak{A}_{o}$ for every classical geometry $E$ on $\mathbb{X}$.

Let us now construct the GNS-representations for $\omega_{E}$. To calculate the Gel'fand ideal, we observe that an element $a$ of $\mathfrak{A}_{o}$ lies in the Gel'fand ideal of $\omega_{E}$ if and only if $\kappa_{E}(a)$ is in the Gel'fand ideal of $\omega_{o}$, where the linear map $\kappa_{E}$ is defined as $\kappa_{E}(f \circ w):=\chi_{E}(w) f \circ w$. The elements of the Gel'fand ideal of $\omega_{o}$ are of the form $a-f(a)$, where $f(a) \in C(\mathcal{A})$ and applying $\kappa_{E}$ does not change this form. It follows that we can choose the image of $\eta_{\omega_{E}}$ takes values in $C(\mathcal{A})$, so we can construct a "Schrödinger" representation analogous to the standard representation of LQG. Moreover, the gauge-variant spin network functions form a dense orthogonal set in $\mathcal{H}_{\omega_{E}}^{o}$ and the trivial spin network function 1 provides a cyclic vacuum vector $\Omega_{E}$. The expectation values for spatial geometric operators (i.e. the elements $w \in \mathcal{W}^{\prime}$ ) are given by the classical values calculated from the geometry $E$ :

$$
\left\langle\Omega_{E}, \pi_{\omega_{E}}(w) \Omega_{E}\right\rangle_{\omega_{E}}=\omega_{E}(w)=\chi_{E}(w) .
$$

We have thus constructed a representation of $\mathfrak{A}_{o}^{\prime}$ where the vacuum expectation values of all geometric operators coincide with classical expectation values for some spatial geometry $E$. Moreover, it follows from direct calculation that $\Omega_{E}$ is an eigenstate of $\mathcal{W}^{\prime}$.

The construction of $\mathfrak{A}_{o}^{\prime}$ required us to restrict ourselves to $\mathcal{W}^{\prime}$ which does not allow to reconstruct all flux operators of LQG. It thus seems at first sight that one may not be able to construct all geometric operators from $\mathcal{W}^{\prime}$. However, since the classical area element contains enough information to reconstruct the metric (at each point one needs to consider six independent conormals), one can use these classical relations to construct quantum geometry operators purely from area operators. We thus see that the restriction to $\mathcal{W}^{\prime}$ does not restrict the number of available geometric operators of LQG. Let us conclude this discussion with the remark that any discussion of the "right" quantization of geometric operators based on the quantization of classical expressions may be completely moot; ultimately geometry is determined by the dynamics of test matter living on it, e.g. by considering the correlation functions of a free field on a "fixed" quantum geometry. The resulting notion of geometry at very short distances can in general not be expected to precisely coincide with the straightforward quantization of a classical relation between geometric operators.

The introduction of a geometric background may at first sight be seen as an obstruction to the implementation of bundle automorphisms (spatial diffeomorphisms and gauge transformations), because for any surface $S$ and any diffeomorphism $\phi$, if diffeomorphisms are implemented unitarily, we have

$$
A_{E}(\phi(S))=\omega_{E}\left(U_{\phi}^{*} A(S) U_{\phi}\right)=\left\langle U_{\phi} \Omega_{E}, A(S) U_{\phi} \Omega_{E}\right\rangle_{\omega_{E}},
$$

where $A_{E}(S)$ denotes the classical area of $S$ given geometry $E$. This can not be realized in $\mathcal{H}_{\omega_{E}}$. The remedy for this problem is to read this as a definition of $U_{\phi} \Omega_{E}$, which implies $U_{\phi} \Omega_{E}=\Omega_{\phi E}$. For this action to be unitary, we have to consider a Hilbert space $\mathcal{H}_{\omega_{E}}^{\phi}$ that is the direct sum of all $\mathcal{H}_{\omega_{E^{\prime}}}$, where $E^{\prime}$ is in the diffeomorphism orbit of $E$. The unitary implementation of gauge transformations works analogously. One ends up with a huge direct sum Hilbert space $\mathcal{H}_{G(E)}$, where $G(E)$ denotes the orbit of $E$ under bundle automorphisms. This is completely analogous to what is done in the case of the holonomy-flux algebra, see (3) and below. Applying the group averaging procedure to mod out the bundle morphisms gives a gauge- and diffeomorphisminvariant Hilbert space $\mathcal{H}_{E}$ that is spanned by gauge-invariant spin network functions that are embedded into a background geometry modulus isomorphisms of the background geometry $G(E)$.

To conclude this section we remark that one can incorporate matter fields in this construction as well. This was explicitly done for a scalar field that could play the role of an inflaton. 


\subsection{Physical interpretation and comparison}

In this section, we would like to make some comments regarding the physical interpretation of the results we have sketched above, as well as the comparison between holonomy-flux algebra and Weyl algebra approach.

Essential geometry. We saw that both the Lie algebra as well as the $C^{*}$-algebra construction lead to a representation of the algebra of LQG where the vacuum vectors $\Omega_{E}$ were eigenvectors of spatial geometry with eigengeometries prescribed by $E$. To interpret the vectors of $\mathcal{H}_{\omega_{E}}$, let us consider how one can reconstruct the "background geometry" $E$ from $v \in \mathcal{H}_{\omega_{E}}$. For this we define the excess of a state $v$ as the smallest countable set of zero and one-dimensional analytic sub-manifolds of the spatial hypersurface, such that the removal of any further zeroor one-dimensional analytic submanifold does not change any of the expectation values of twodimensional geometric operators. Now define the essential geometry of a state $v$ as the smooth geometry $E$ that is reconstructed from the expectation values of the two-dimensional geometric operators after the removal of the excess of $v$. Notice that the excess of a state $v$ removes all LQG excitations, since these are supported at most on a countable number of piecewise analytic edges; the resulting essential geometry is thus precisely the geometry $E$ that we used to construct $\omega_{E}$.

Background coupling. Since operators that measure the essential geometry can be defined solely from operators that are already in $\mathcal{W}^{\prime}$, it is natural to include these "background"operators. These operators $\tilde{E}_{\tau}(x),|\tilde{E}|(x)$ act on a state $T \Omega_{E_{o}}$ with essential geometry $E_{o}$ as $\left(\tilde{E}_{\tau}(x),|\tilde{E}|(x)\right) T \Omega_{E_{o}}=\left(E_{o, \tau}(x),\left|E_{o}\right|(x)\right) T \Omega_{E_{o}}$. This is redundant if one considers a representation on one summand $\mathcal{H}_{\omega_{E}}$, since these operators are proportional to the unit operator and can thus be identified with multiplication by numbers. However, if one considers a representation on a direct sum $\mathcal{H}_{G(E)}$ then the background geometry operators are not proportional to the unit operator. Moreover, the transformation properties of these operators under bundle automorphisms is induced. Let us now consider a gauge-invariant operator $\hat{O}$ constructed from holonomy matrix elements and background geometry operators and consider the action on $\Omega_{E}$ as an element of $\mathcal{H}_{G(E)}$. Let us now apply the group averaging procedure for the bundle automorphisms to the resulting element $\hat{O} \Omega_{E}$. Due to the nontrivial transformation properties of the background geometry operators, one can not expand the resulting vector in gauge-invariant spin network functions, but one has to allow for gauge-invariant couplings of the spin network to the background geometry as well. We will hint towards a possible physical interpretation of this possible background coupling when we discuss the nondegenerate vacuum representations in the context of effective field theory.

Hamiltonian constraint quantization. It is interesting to see whether the Hamiltonian constraint can be quantized in the new representations. On the one hand, one can try the standard techniques. In the $\mathfrak{H F}$ approach, this can be carried out with a surprising result. In the standard approach [29], a regularization of the Hamiltonian constraint is defined on $\mathcal{H}_{\mathrm{AL}}$ in terms of holonomies and the volume operator $V^{\mathrm{vac}}$. It can be checked that this construction can be repeated for each of the $\mathcal{H}_{E^{(0)}}$ and hence on $\mathcal{H}_{G\left(E^{(0)}\right)}$. Thus the regularizations of the Hamilton constraint are well defined, and solutions can be sought. The action of $V$ is certainly modified in the new representations, but the volume operator only enters in terms of commutators $[h, V]$ with holonomies or $\left[H_{\text {euc }}, V\right]$ with the Euclidean Hamiltonian constraint. These commutators are not modified. Therefore

$$
\left(f, E^{(0)} \mid \widehat{H}(N)=0 \Longleftrightarrow\left(f, E^{\prime(0)} \mid \widehat{H}(N)=0\right.\right.
$$

for $E^{(0)}, E^{\prime(0)}$ not necessarily gauge related. In particular, any solution over the standard vacuum is a solution over non-trivial backgrounds. The meaning of this observation is not clear. 
On the other hand, with a non-degenerate background one has more possibilities available for the quantization of the Hamilton constraint. Essentially, the inverse volume problem is gone, and the quantization of the functional dependence on $E$ in the constraint gets trivialized. The problems with quantizing curvature, on the other hand, remain. How these observations can be used for a new quantization of the constraint is currently under study.

$\mathfrak{H} \mathfrak{F}$ vs. $\mathfrak{A}$. Although the holonomy-flux representation theory together with correct domain assumptions and Fleischhack's Weyl algebra are closely related, there is a subtle difference. It arises, because the holonomy-flux commutation relations do not close as a Lie algebra, because the commutator of two flux operators (i.e. through a natural surface) is proportional to a quasiflux (i.e. through a quasi-surface). Regularity of the representation of the $C^{*}$-algebra ensures that one has a representation of the holonomy-flux algebra on a dense domain. The holonomyflux algebra which closes to a particular Lie algebra involving quasi flux operators. However, there are more Lie algebras that contain the physically important holonomy-flux algebra. One can in particular relax the assumption that commutators of the physically dubious quasi fluxes are required to reproduce their classical Poisson brackets. One obtains a richer representation theory if one relaxes this assumption. This is the technical reason why the nondegenerate representations can only be defined for the restricted algebra $\mathcal{W}^{\prime}$ and not all of $\mathcal{W}$, while the analogous restriction is not necessary in the Lie-algebraic setting.

\section{Outlook}

The F/LOST-uniqueness result was a boost for the development of LQG, because it basically taught us that there is a unique diffeomorphism-invariant kinematic ground state. Let us thus justify the nondegenerate representations by considering possible applications:

1. Effective Field Theory. Effective field theory is a very valuable tool for the investigation of low-energy effects of a quantum field theory, that can be used even if the high energy theory is unknown. Representations with nondegenerate background geometry can be used to study effective field theory of LQG fluctuations in a semiclassical background. The nondegenerate background geometry is particularly useful since its presence allows one to use e.g. the background metric to construct coarse graining operations for LQG fluctuations. The idea is that loop structures that are smaller than a given scale w.r.t. the background metric are integrated out and are effectively described as shift in the background metric after a coarse graining step. This may open the door for the application of Wilsonian renormalization methods to LQG. Such a procedure would interpolate between "bumpy" Loop Quantum Geometries in the UV and smooth background geometries in the IR. This avoids many of the problems associated with the geometric interpretation of spin network states.

The asymptotic safety program by Reuter and collaborators does not have this problem, because it is formulated entirely in a continuum field theory setting. The idea behind this program is to track the change of the effective action as one integrates out quantum fluctuations at a momentum scale $k$. This program has accumulated nonperturbative evidence for the existence a non-Gaussian fixed point and it follows from simple dimensional analysis that the effective dimension at the non-Gaussian fixed point is 2 while the effective dimension at low energies is 4. This suggested that the fundamental theory should have 1-dimensional excitations of space, which matches with spin network excitation in LQG. This has lead to speculations that LQG (with degenerate vacuum) may be able to provide the fundamental description of the fixed point theory.

A concrete implementation of this scenario is however elusive. A main problem is that a coarse graining procedure that starts with a "fundamental" Ashtekar-Lewandowski state and ends up with a state with effective nondegenerate background geometry is missing. This is partly due to the aforementioned problem of finding the continuum geometry that "best approximates" 
the loop geometry while smoothing out short distance bumpiness. It is thus at the moment not possible to depart from LQG (with degenerate vacuum) in the UV and end up with an effective continuum theory in the IR.

Let us now return to background couplings in light of an effective field theory interpretation. Consider a spin network state whose graph is a regular lattice with small nonvanishing spin quantum numbers on all edges of the lattice plus a few nonlocal links with nonvanishing spin quantum numbers. Let us furthermore assume a coarse graining procedure that absorbs the entire lattice as a shift of the background geometry, so only the nonlocal links are left after coarse graining. It has been suggested that the endpoints of nonlocal links should appear like matter to a local observer. If this assertion holds then one should interpret the background couplings as Loop Quantum Geons.

2. Reinterpretation of Loop Quantum Cosmology. Loop Quantum Cosmology is generally understood as a toy model that incorporates important features of LQG, but a precise mathematical relation is not established. Nondegenerate representations allow for a different interpretation where standard Loop Quantum Cosmology is interpreted as the dynamics of a homogeneous background geometry. This could be used to couple arbitrary Loop Quantum fluctuations to standard Loop Quantum Cosmology.

3. Further Generalizations. The main technical problem in constructing the above states is to prove positivity of the GNS functional. The positivity proof for the GNS functionals allows for several generalizations, which are not explored. It may be particularly useful to investigate states that are invariant under certain flows. This may avoid "quantization" by providing a completely mathematical selection criterion for Loop Quantum dynamics as unitarily implementable flows.

Let us conclude this review with a provocative thought: "The nondegenerate representations may hint what the essence of quantum gravity is." Let us explain what we mean: Many of the nice features of LQG are due to the fact that the kinematic Hilbert space can be constructed as the closure of a set of normalized and mutually orthogonal eigenstates of spatial quantum ${ }^{7}$ geometry. E.g. the unitarity of the natural action of spatial diffeomorphisms as well as the "discreteness" of spatial geometric operators is a direct consequence. This is particularly transparent in the nondegenerate representations. It is thus not unreasonable to attempt new approaches to quantum gravity that start with a Hilbert space that is the completion of mutually orthogonal normalized eigenstates of spatial geometry and implement exponentiated momentum operators as pull-backs under translations in the geometrodynamic configuration space.

\section{Acknowledgements}

HS gratefully acknowledges partial support through the Spanish MICINN Project No. FIS200806078-C03-03. Research at the Perimeter Institute is supported in part by the Government of Canada through NSERC and by the Province of Ontario through MEDT.

\section{References}

[1] Ashtekar A., Lewandowski J., Differential geometry on the space of connections via graphs and projective limits, J. Geom. Phys. 17 (1995), 191-230, hep-th/9412073.

[2] Ashtekar A., Lewandowski J., Projective techniques and functional integration for gauge theories, J. Math. Phys. 36 (1995), 2170-2191, gr-qc/9411046.

[3] Ashtekar A., Lewandowski J., Quantum theory of geometry. I. Area operators, Classical Quantum Gravity 14 (1997), A55-A81, gr-qc/9602046.

\footnotetext{
${ }^{7}$ We include the attribute quantum, because of the noncommutativity of Loop Quantum Geometry operators on the gauge-invariant Hilbert space.
} 
[4] Ashtekar A., Lewandowski J., Relation between polymer and Fock excitations, Classical Quantum Gravity 18 (2001), L117-L127, gr-qc/0107043.

[5] Ashtekar A., Lewandowski J., Marolf D., Mourão J., Thiemann T., Quantization of diffeomorphism invariant theories of connections with local degrees of freedom, J. Math. Phys. 36 (1995), 6456-6493, gr-qc/9504018.

[6] Ashtekar A., Rovelli C., Smolin L., Weaving a classical metric with quantum threads, Phys. Rev. Lett. 69 (1992), 237-240, hep-th/9203079.

[7] Bahr B., Thiemann T., Gauge-invariant coherent states for loop quantum gravity. I. Abelian gauge groups, Classical Quantum Gravity 26 (2009), 045011, 22 pages, arXiv:0709.4619.

[8] Bahr B., Thiemann T., Gauge-invariant coherent states for loop quantum gravity. II. Non-Abelian gauge groups, Classical Quantum Gravity 26 (2009), 045012, 45 pages, arXiv:0709.4636.

[9] Bianchi E., Magliaro E., Perini C., Coherent spin-networks, Phys. Rev. D 82 (2010), 024012, 7 pages, arXiv:0912.4054.

[10] Dziendzikowski M., Okolów A., New diffeomorphism invariant states on a holonomy-flux algebra, Classical Quantum Gravity 27 (2010), 225005, 24 pages, arXiv:0912.1278.

[11] Fleischhack C., Construction of generalized connections, math-ph/0601005.

[12] Fleischhack C., Irreducibility of the Weyl algebra in loop quantum gravity, Phys. Rev. Lett. 97 (2006), 061302, 4 pages, math-ph/0407006.

[13] Fleischhack C., Representations of the Weyl algebra in quantum geometry, Comm. Math. Phys. 285 (2009), 67-140, math-ph/0407006.

[14] Freidel L., Livine E.R., U(N) coherent states for loop quantum gravity, J. Math. Phys. 52 (2011), 052502, 21 pages, arXiv:1005.2090.

[15] Koslowski T.A., Cosmological sectors in loop quantum gravity, Ph.D. thesis, Universität Würzburg, 2008.

[16] Koslowski T.A., Dynamical quantum geometry (DQG programme), arXiv:0709.3465.

[17] Lewandowski J., Volume and quantizations, Classical Quantum Gravity 14 (1997), 71-76, gr-qc/9602035.

[18] Lewandowski J., Okolów A., Sahlmann H., Thiemann T., Uniqueness of diffeomorphism invariant states on holonomy-flux algebras, Comm. Math. Phys. 267 (2006), 703-733, gr-qc/0504147.

[19] Okolów A., Lewandowski J., Automorphism covariant representations of the holonomy-flux *-algebra, Classical Quantum Gravity 22 (2005), 657-679, gr-qc/0405119.

[20] Okolów A., Lewandowski J., Diffeomorphism covariant representations of the holonomy-flux $\star$-algebra, Classical Quantum Gravity 20 (2003), 3543-3567, gr-qc/0302059.

[21] Oriti D., Sindoni L., Toward classical geometrodynamics from the group field theory hydrodynamics, New J. Phys. 13 (2011), 025006, 44 pages, arXiv:1010.5149.

[22] Sahlmann H., On loop quantum gravity kinematics with a non-degenerate spatial background, Classical Quantum Gravity 27 (2010), 225007, 17 pages, arXiv:1006.0388.

[23] Sahlmann H., Some results concerning the representation theory of the algebra underlying loop quantum gravity, J. Math. Phys. 52 (2011), 012502, 9 pages, gr-qc/0207111.

[24] Sahlmann H., When do measures on the space of connections support the triad operators of loop quantum gravity?, J. Math. Phys. 52 (2011), 012503, 14 pages, gr-qc/0207112.

[25] Sahlmann H., Thiemann T., Irreducibility of the Ashtekar-Isham-Lewandowski representation, Classical Quantum Gravity 23 (2006), 4453-4471, gr-qc/0303074.

[26] Sahlmann H., Thiemann T., On the superselection theory of the Weyl algebra for diffeomorphism invariant quantum gauge theories, gr-qc/0302090.

[27] Thiemann T., Complexifier coherent states for quantum general relativity, Classical Quantum Gravity 23 (2006), 2063-2117, gr-qc/0206037.

[28] Thiemann T., Gauge field theory coherent states (GCS). I. General properties, Classical Quantum Gravity 18 (2001), 2025-2064, hep-th/0005233.

[29] Thiemann T., Quantum spin dynamics (QSD), Classical Quantum Gravity 15 (1998), 839-873, gr-qc/9606089.

[30] Varadarajan M., Fock representations from U(1) holonomy algebras, Phys. Rev. D 61 (2000), 104001, 13 pages, gr-qc/0001050.

[31] Varadarajan M., Towards new background independent representations for loop quantum gravity, Classical Quantum Gravity 25 (2008), 105011, 17 pages, arXiv:0709.1680. 